Research Article

\title{
Beamforming and Interference Cancellation in D2D Random Network
}

\author{
Langtao $\mathrm{Hu}^{1,2}$ and Chaowei Yuan ${ }^{1}$ \\ ${ }^{1}$ Beijing University of Posts and Telecommunications, Beijing 100876, China \\ ${ }^{2}$ Anqing Normal University, Anqing 246011, China \\ Correspondence should be addressed to Langtao Hu; 122634998@qq.com
}

Received 15 January 2015; Revised 3 March 2015; Accepted 4 March 2015

Academic Editor: Heng-Tung Hsu

Copyright (C) 2015 L. Hu and C. Yuan. This is an open access article distributed under the Creative Commons Attribution License, which permits unrestricted use, distribution, and reproduction in any medium, provided the original work is properly cited.

Device-to-Device (D2D) communication is an important proximity communication technology. We model the hybrid network of cellular and D2D communication with stochastic geometry theory. In the network, cellular base stations are deployed with multiantennas. Two transmission strategies including beamforming and interference cancellation are proposed to boost system achievable rate in this paper. We derive analytical success probability and rate expression in these strategies. In interference cancellation strategy, we propose the partical BS transmission degrees of freedom (dofs) that can be used to cancel its D2D users (DUEs) interferences around the BS or to boost the desired signal power of associated cellular (CUE). In order to maximize the total area spectral efficiency (ASE), the BS transmission degrees of freedom are allocated according to proper interference cancellation radius around the BS. Monte Carlo simulations are performed to verify our analytical results, and two transmission strategies are compared.

\section{Introduction}

Device-to-Device (D2D) communication is an important proximity communication technology, which has been in standard process of LTE-advanced system and it is a key technology for the future hybrid networks. With the development of mobile internet, the cellular network is not able to meet the requirements for the future localizing applications and D2D technology comes to an important complement for it [1-3]. The performance of wireless communication can be analyzed accurately by stochastic geometry theory. Traditional model has Wyner model or hexagonal grid [4]. The Wyner model or the hexagonal grid can be evaluated by system-level simulations. However, both the scalability and the accuracy of grid model were questionable in the context of network heterogeneity [4-6]. An alternative is to model the locations of sites as random and drawn from a spatial stochastic process, such as the Poisson point process (PPP), which has been confirmed as accurate as the grid model [5]. This stochastic model has been used recently in [7] to analyze success probability and average rate of heterogeneous network.
Reference [1] has studied spectrum sharing and derived analytical rate expressions for $\mathrm{D} 2 \mathrm{D}$ communication in cellular networks by stochastic geometry theory and compared with signal to noise plus interference ratios (SINR) distribution using the hexagonal model by Monte Carlo. In [8], the spatial distribution of transmit powers and SINR are studied, and cumulative distribution function (CDF) of the transmit power and SINR have analytically been derived for a D2D network employing power control. In [9], mode selection and power control have been presented for underlay D2D communication in cellular networks, in which the proposed mode selection scheme for a user accounted for both the D2D link distance and cellular link distance (i.e., distance between the CUE and the BS). In [10], the small-scale fading experienced in the D2D direct link is modeled as Rician distribution.

Most of the previous works, for example [7-10], study results were based on single antenna deployed at BS in cellular network by stochastic geometry theory. Multiple antenna techniques are already relatively mature, and many standardization activities clearly indicate that multiantenna techniques and hybrid network will coexist and complement 


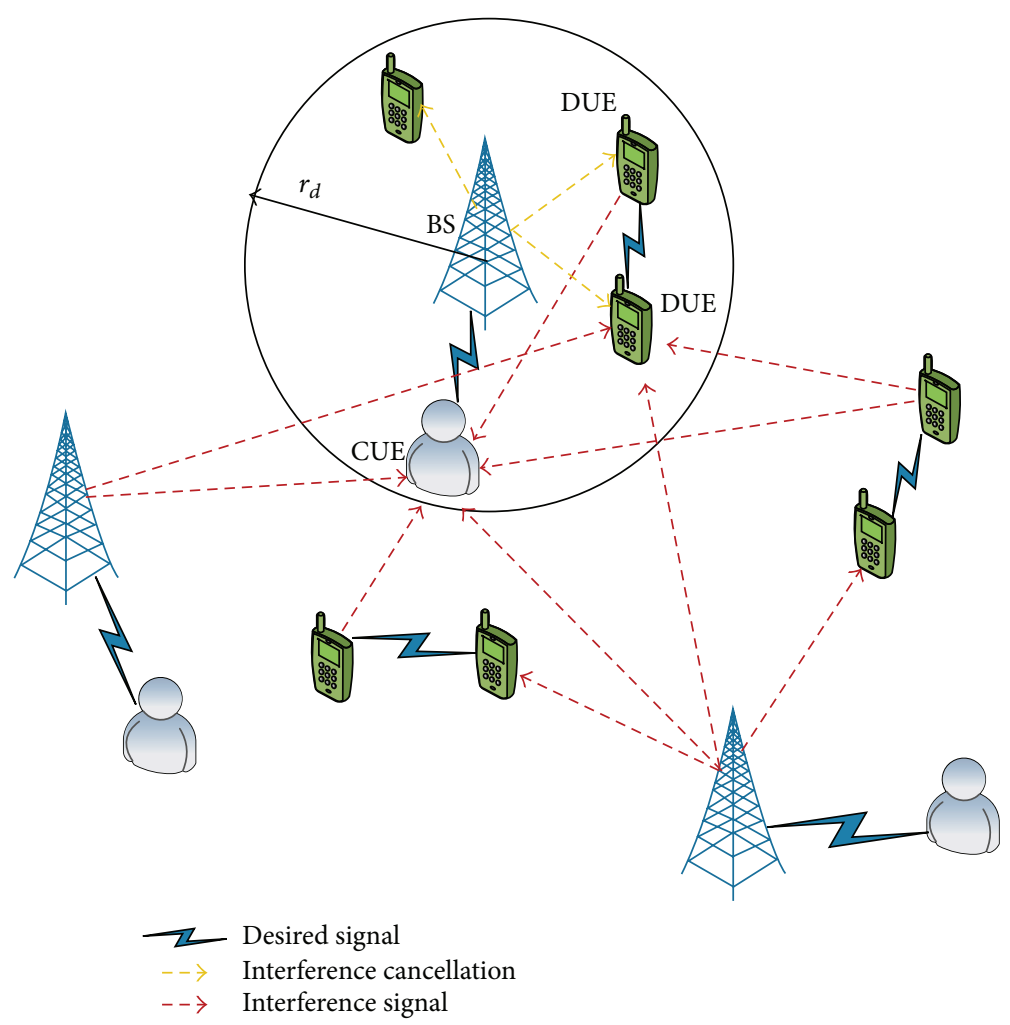

FIGURE 1: Cellular and D2D communication model. $r_{d}$ is interference cancellation radius in interference cancellation strategy.

each other in the future wireless networks and should not be studied in isolation, as has been typically done in the literature [11]. Multiple antenna techniques have many significant features [12-14], such as using precoding design for interference cancellation or using beamforming design for boosting diversity gain. In random network, average achievable rate and reliability can be improved by the multiple antenna techniques [15-17]. In this paper, we model the hybrid network of cellular and D2D communication with stochastic geometry theory. In the network, cellular base stations are deployed with multiantennas. We analyze two primary performance measures: success probability and average achievable rate expression. The rate performance of beamforming and interference cancellation strategies is compared.

The rest of the paper is organised as follows. In Section 2, we introduce the system model. In Section 3, the success probability and average rate performance of beamforming strategy are investigated. In Section 4, the success probability and average rate performance of interference cancellation strategy are investigated. In Section 5, numerical simulation and analysis are discussed to verify these results. A conclusion is drawn in Section 6.

Notation. Let a denote a vector. Transpose and conjugate transpose are denoted by $T$ and $H$. The expectation of function $f(x)$ with respect to $x$ is denoted as $E[f(x)]$. The Laplace transform of $f(x)$ is denoted by $L_{f}(s)$. A circularly symmetric complex Gaussian random variable $x$ with zero mean and variance $\sigma^{2}$ is denoted as $x \sim \mathbb{C N}\left(0, \sigma^{2}\right)$. A Chi-square distributed random variable $x$ with $N$ degree of freedom is denoted by $x \sim \chi^{2}(N)$. An exponential distributed random variable with mean 1 is denoted by $x \sim \exp (1)$. Let $I_{1}$ be a set and let $I_{2}$ be a subset of $I_{1}$; then $I_{1} \backslash I_{2}$ denotes the set of elements of $I_{1}$ that do not belong to $I_{2}$.

\section{System Model}

We consider a downlink hybrid network of cellular and D2D communication [1], as shown in Figure 1. The locations of base stations (BSs) are deployed as a PPP [5] $\phi_{B}=$ $\left\{\Gamma_{B n}, n \in \mathbb{N}\right\}$ with intensity $\lambda_{B}$ and constant transmission power $p_{B}$. Similarly, the cellular users locations are modeled as a PPP $\phi_{U}=\left\{\Gamma_{U n}, n \in \mathbb{N}\right\}$ with intensity $\lambda_{U}$. The locations of the D2D users are assumed to follow a PPP $\phi_{D}=$ $\left\{\Gamma_{D n}, n \in \mathbb{N}\right\}$ with intensity $\lambda_{D}$ and constant transmission power $p_{D}$. We assume the whole bandwidth $W$ is divided into $V$ subchannels. All the subchannels are available for BSs and D2D. Each D2D transmitter may randomly and independently access the subchannel. Each BS is configured with $N$ antennas. Each CUE and DUE is configured with single antenna. The downlink channel is composed of path large-scale attenuation and fading for both cellular networks and D2D communication. Large-scale attenuation is modeled as the standard pathloss propagation represented as $d_{T R}^{-a / 2}$, where $a$ is path-loss exponent and $d_{T R}$ is distance between transmitter $T$ and receiver $R$. $T$ may be BS or DUE transmitter, and $R$ may be DUE receiver or CUE receiver. 
Each CUE is associated with the nearest BS. Therefore, the probability density function (PDF) of the distance $d_{B C}$ can be derived as $f_{d_{B C}}(r)=\exp \left(-\pi \lambda_{B} r^{2}\right) 2 \pi \lambda_{B} r$ according to the null probability of a $2 \mathrm{D}$ Poisson process [10]. Each DUE transmitter and its DUE receiver have a fixed distance of $d_{D D}$. Meanwhile, Rayleigh fading is assumed for the BS-CUE, DUE-CUE, BS-DUE, and DUE-DUE links. We consider the interference limited regime; that is, noise power is negligible compared to the interference power [10]. In the following, we will characterize the performance of beamforming and interference cancellation strategies.

\section{Beamforming Strategy}

With multiple antennas, the BS chooses to increase its own cellular user signal power by performing beamforming. $y_{C 0}$ and $y_{D 0}$ are the received signal at the typical CUE $\left(R_{C 0}\right)$ and typical DUE receiver $\left(R_{D 0}\right)$, respectively,

$$
\begin{aligned}
y_{C 0}= & \sum_{n: \Gamma_{B n} \in \phi_{B}} \sqrt{p_{B}} d_{B C, n}^{-a / 2} \mathbf{q}_{0 n} \mathbf{u}_{n} x_{B n} \\
& +\sum_{n: \Gamma_{D n} \in \phi_{D}} \sqrt{p_{D}} d_{D C, n}^{-a / 2} f_{0 n} x_{D n} \\
y_{D 0}= & \sum_{n: \Gamma_{D n} \in \phi_{D}} \sqrt{p_{D}} d_{D D, n}^{-a / 2} h_{0 n} x_{D n} \\
& +\sum_{n: \Gamma_{B n} \in \phi_{B}} \sqrt{p_{B}} d_{B D, n}^{-a / 2} \mathbf{g}_{0 n} \mathbf{u}_{n} x_{B n}
\end{aligned}
$$

where $\mathbf{q}_{0 n} \in \mathbb{C}^{1 \times N}$ is the channel between BS in $\phi_{B}$ and $R_{C 0} \cdot f_{0 n}$ is the channel between $R_{C 0}$ and DUE transmitter in $\phi_{D} \cdot h_{0 n}$ is the channel between $R_{D 0}$ and DUE transmitter in $\phi_{D} \cdot \mathbf{g}_{0 n} \in \mathbb{C}^{1 \times N}$ is the channel between $R_{D 0}$ and BS in $\phi_{B} . \mathbf{u}_{n} \in \mathbb{C}^{N \times 1}$ is the beamformer used by the $n$th transmitter. $x_{B n}$ and $x_{D n}$ are the data signals transmitted from BS and DUE, respectively. $x_{B n}$ and $x_{D n}$ are $\sim \mathbb{C N}(0,1)$. The signal to interference ratio (SIR) in $R_{D 0}$ with beamforming strategy is

$$
\operatorname{SIR}_{D_{\mathrm{BF}}}=\frac{P_{D} d_{D D}^{-a}\left|h_{00}\right|^{2}}{I_{D_{\mathrm{BF}}}}
$$

where $I_{D_{\mathrm{BF}}}$ denotes the total interference, $I_{D_{\mathrm{BF}, 1}}$ denotes the interference from DUEs, and $I_{D_{\mathrm{BF}, 2}}$ denotes the interference from BSs. $\widetilde{I}_{D_{\mathrm{BF}, 1}}$ and $\widetilde{I}_{D_{\mathrm{BF}, 2}}$ are simple transform of $I_{D_{\mathrm{BF}, 1}}$ and $I_{D_{\mathrm{BF}, 2}}$ by normalizing the desired signal power, respectively,

$$
\begin{aligned}
I_{D_{\mathrm{BF}}}= & \underbrace{\sum_{n: \Gamma_{D n} \in \phi_{D} \backslash\left\{\Gamma_{D 0}\right\}} p_{D} d_{D D, n}^{-a}\left|h_{0 n}\right|^{2}}_{I_{D_{\mathrm{BF}, 1}}} \\
& +\underbrace{}_{I_{D_{\mathrm{BF}, 2}} \sum_{n: \Gamma_{B n} \in \phi_{B}} p_{B} d_{B D, n}^{-a}\left|\mathbf{g}_{0 n} \mathbf{u}_{n}\right|^{2}}
\end{aligned}
$$

$$
\begin{gathered}
\widetilde{I}_{D_{\mathrm{BF}, 1}}=\sum_{n: \Gamma_{D n} \in \phi_{D} \backslash\left\{\Gamma_{D 0}\right\}} \frac{d_{D D, n}^{-a}\left|h_{0 n}\right|^{2}}{d_{D D}^{-a}}, \\
\widetilde{I}_{D_{\mathrm{BF}, 2}}=\sum_{n: \Gamma_{B n} \in \phi_{B}} \frac{d_{B D, n}^{-a}\left|\mathbf{g}_{0 n} \mathbf{u}_{n}\right|^{2}}{d_{D D}^{-a} p_{D} / p_{B}} .
\end{gathered}
$$

The signal to interference ratio (SIR) in $R_{C 0}$ with beamforming strategy is

$$
\operatorname{SIR}_{C_{\mathrm{BF}}}=\frac{P_{B} d_{B C}^{-a}\left|\mathbf{q}_{00} \mathbf{u}_{0}\right|^{2}}{I_{C_{\mathrm{BF}}}},
$$

where $I_{C_{\mathrm{BF}}}$ denotes the total interference at $R_{C 0}$ and $I_{C_{\mathrm{BF}, 1}}$ denotes the interference from BSs. $I_{C_{\mathrm{BF}, 2}}$ denotes the interference from DUEs and $\widetilde{I}_{C_{\mathrm{BF}, 1}}$ and $\widetilde{I}_{C_{\mathrm{BF}, 2}}$ are simple transform of $I_{C_{\mathrm{BF}, 1}}$ and $I_{C_{\mathrm{BF}, 2}}$ by normalizing the desired signal power, respectively,

$$
\begin{gathered}
I_{C_{\mathrm{BF}}}=\underbrace{\sum_{n: \Gamma_{B n} \in \phi_{B} \backslash\left\{\Gamma_{B 0}\right\}} p_{B} d_{B C, n}^{-a}\left|\mathbf{q}_{0 n} \mathbf{u}_{n}\right|^{2}}_{I_{C_{\mathrm{BF}, 1}}} \\
+\underbrace{\sum_{C_{\mathrm{BF}, 2}}}_{\sum_{n: \Gamma_{D n} \in \phi_{D}} p_{D} d_{D C, n}^{-a}\left|f_{0 n}\right|^{2},} \\
\widetilde{I}_{C_{\mathrm{BF}, 1}}=\sum_{n: \Gamma_{B n} \in \phi_{B} \backslash\left\{\Gamma_{B 0}\right\}} \frac{d_{B C, n}^{-a}\left|\mathbf{q}_{0 n} \mathbf{u}_{n}\right|^{2}}{d_{B C}^{-a}}, \\
\widetilde{I}_{C_{\mathrm{BF}, 2}}=\sum_{n: \Gamma_{D n} \in \phi_{D}} \frac{d_{D C, n}^{-a}\left|f_{0 n}\right|^{2}}{d_{B C}^{-a} p_{D} / p_{B}} .
\end{gathered}
$$

According to the BF criterion via receiving power maximization, the precoding vector $\mathbf{u}_{n}$ should align with the same direction as the channel itself $\mathbf{u}_{n}=\mathbf{q}_{o n} /\left\|\mathbf{q}_{o n}\right\|^{2}$; then the signal power at the $R_{C 0}$ is $\left|\mathbf{q}_{00} \mathbf{u}_{0}\right|^{2} \sim \chi^{2}(2 N)$ and the interference power at the $R_{C 0}$ from other BS is $\left|\mathbf{q}_{0 n} \mathbf{u}_{n}\right|^{2} \sim \chi^{2}(2)$ as [15]. Similarly, $\left|\mathbf{g}_{0 n} \mathbf{u}_{0}\right|^{2} \sim \chi^{2}(2)$. Because $f_{0 n} \sim \mathbb{C N}(0,1)$, we have $\left|f_{0 n}\right|^{2} \sim \chi^{2}(2)$.

A performance metric of interest in this study is the success transmission probability of BS $P_{\text {Suc.C }}\left(\beta_{C}\right)$ with respect to a predefined SIR threshold $\beta_{C}$, similarly, the success transmission probability of D2D network $P_{\text {Suc. } D}\left(\beta_{D}\right)$ with respect to a predefined SIR threshold $\beta_{D}$. The D2D and cellular network success probability are given in Theorem 1 .

Theorem 1. For the cellular network underlay with D2D communication, the cellular BSs are configured with $N$ antennas for beamforming strategy. The cellular and D2D success probability are given by

$$
\begin{gathered}
P_{\text {Suc. } D_{B F}}\left(\beta_{D}\right)=\exp \left(-C_{d 1} \beta_{D}^{2}\right), \\
P_{\text {Suc. } C_{B F}}\left(\beta_{C}\right)=\frac{\pi \lambda_{B}}{A_{0}}+\sum_{k=1}^{N-1} \sum_{j=1}^{k} \frac{(-1)^{k}}{k !} \pi \lambda_{B} A_{j}^{k} \frac{\Gamma(j+1)}{\left(A_{0}\right)^{j+1}},
\end{gathered}
$$


where

$$
\begin{gathered}
C_{d 1}=\pi c(a) d_{D D}^{a / 2}\left(\lambda_{D}+\lambda_{B}\left(\frac{p_{B}}{p_{D}}\right)^{2 / a}\right), \\
A_{0}=\left.\left(C_{d 2}^{\prime} s^{2 / a}+\pi \lambda_{B}\right)\right|_{s=\beta_{c}}=C_{d 2}^{\prime}\left(\beta_{C}\right)^{2 / a}+\pi \lambda_{B}, \\
A_{j}^{k}=\left.\frac{\beta_{j}^{k}}{j !}\left(C_{d 2}^{\prime} s^{2 / a}\right)^{j}\right|_{s=\beta_{c}}=\frac{\beta_{j}^{k}}{j !}\left(C_{d 2}^{\prime}\left(\beta_{C}\right)^{2 / a}\right)^{j}, \\
\beta_{j}^{k}=\sum_{i=1}^{j}(-1)^{j}\left(\begin{array}{l}
j \\
i
\end{array}\right)\left(\frac{2 i}{a}\right)_{k}, \quad j=1, \ldots, k, \\
(x)_{k}=(x)(x-1) \cdots(x-k+1) .
\end{gathered}
$$

Proof. See Appendix A.

Theorem 2. For the cellular network underlay with D2D communication, the cellular BSs are configured with $N$ antennas for beamforming strategy. The cellular and D2D average achievable rate in a shared subchannel are given by

$$
\begin{gathered}
R_{D_{B F}}=\Lambda\left(C_{d 1}\right), \\
R_{C_{B F}}=\int_{0}^{\infty} \int_{z=0}^{\infty} \frac{\sum_{k=0}^{N-1}(1 / k !)(-z)^{k} Q(z, k)}{1+z} \\
\cdot 2 \pi \lambda_{B} r \exp \left(-\pi \lambda_{B} r^{2}\right) d z d r,
\end{gathered}
$$

where

$$
\begin{gathered}
\Lambda(x)=\pi \sin x-2 \sin x \operatorname{Si}(x)-2 \cos x \operatorname{Ci}(x), \\
\operatorname{Si}(x)=\int_{0}^{x} \frac{\sin t}{t} d t, \\
\operatorname{Ci}(x)=-\int_{x}^{\infty} \frac{\cos t}{t} d t,
\end{gathered}
$$

$$
\begin{aligned}
& Q(s, k) \\
& =\frac{d L_{I_{C_{B F}}}(s)}{d s^{k}} \\
& = \begin{cases}\exp \left(-C_{d 2} s^{2 / a}\right), & k=0 \\
\left(-\frac{1}{s}\right)^{k} \exp \left(-C_{d 2} s^{2 / a}\right) \sum_{j=1}^{k} \frac{\beta_{j}^{k}}{j !}\left(C_{d 2} s^{2 / a}\right)^{j}, & k \neq 0,\end{cases} \\
& C_{d 2}=\pi c(a) d_{B C}^{2}\left(\lambda_{B}+\lambda_{D}\left(\frac{p_{D}}{p_{B}}\right)^{2 / a}\right) .
\end{aligned}
$$

Proof. See Appendix B.

It is difficult to derive the closed form expressions by directly integrating in (14). Based on this expression, a practical case of $a=4$ and known $d_{B C}$ is applied to further derive the closed form of $R_{C_{\mathrm{BF}}}$, given by

$$
\begin{aligned}
& R_{C_{\mathrm{BF}} \mid d_{B C}}=E\left[\log (1+\xi) \mid d_{B C}\right] \\
& =\Lambda\left(C_{d 2}\right) \\
& +\sum_{k=1}^{N-1} \sum_{j=1}^{k} \frac{\beta_{j}^{k}(-1)^{k}}{j !} G_{1,3}^{3,1}\left(\begin{array}{c}
-\frac{j}{2} \\
-\frac{j}{2}, 0, \frac{1}{2}
\end{array} \mid \frac{C_{d 2}^{2}}{4}\right),
\end{aligned}
$$

where $G_{p, q}^{m, n}\left(\begin{array}{l}a_{1}, \ldots, a_{n}, a_{n+1}, \ldots, a_{p} \\ b_{1}, \ldots, b_{m}, b_{m+1}, \ldots, b_{q}\end{array} \mid z\right)$ is Meijer-G function [10]. The proof of Expression (16) is given by

$$
\begin{aligned}
E & {\left[\log (1+\xi) \mid d_{B C}\right] } \\
& =\int_{x=0}^{\infty} \log (1+x) f_{\xi}(x) d x \\
& =\int_{x=0}^{\infty} \int_{z=0}^{\infty} \frac{1}{1+z} f_{\xi}(x) d z d x \\
& =\int_{z=0}^{\infty} \frac{1-F_{\xi}(z)}{1+z} d z \\
& =\int_{z=0}^{\infty} \frac{\sum_{k=0}^{N-1}(1 / k !)(-z)^{k} Q(z, k)}{1+z} d z \\
& =\Lambda\left(C_{d 2}\right)+\sum_{k=1}^{N-1} \sum_{j=1}^{k} \frac{\beta_{j}^{k}(-1)^{k}}{j !} G_{1,3}^{3,1}\left(\begin{array}{c}
-\frac{j}{2} \\
-\frac{j}{2}, 0, \frac{1}{2}
\end{array} \text { । } \frac{C_{d 2}^{2}}{4}\right) .
\end{aligned}
$$

\section{Interference Cancellation Strategy}

When the Interference cancellation strategy is exploited at the BS, the strategy employs a partial zero forcing (PZF) beamforming vector $[18,19]$. It was found to be amenable to analysis and to explicitly balance interference cancellation and boosting of the desired signal power. With PZF, the BS uses $L$ degrees of freedom to cancel its $L$ DUE interferences inside a circle of radius $r_{d}$ centered around the BS and uses the remaining $N-L$ degrees of freedom to transmit the desired signal to its associated CUE receiver. In the following, we will characterize the performance of system based on interference cancellation (i.e., PZF) strategy. $y_{C 0}$ and $y_{D 0}$ are the received signal at the typical CUE $\left(R_{C 0}\right)$ and typical DUE receiver $\left(R_{D 0}\right)$, respectively. One has

$$
\begin{aligned}
y_{C 0}= & \sum_{n: \Gamma_{B n} \in \phi_{B}} \sqrt{p_{B}} d_{B C, n}^{-a / 2} \mathbf{q}_{0 n} \mathbf{u}_{n} x_{B n} \\
& +\sum_{n: \Gamma_{D n} \in \phi_{D}} \sqrt{p_{D}} d_{D C, n}^{-a / 2} f_{0 n} x_{D n},
\end{aligned}
$$




$$
\begin{aligned}
y_{D 0}= & \sum_{n: \Gamma_{D n} \in \phi_{D}} \sqrt{p_{D}} d_{D D, n}^{-a / 2} h_{0 n} x_{D n} \\
& +\sum_{n: \Gamma_{B n} \in \phi_{B} \backslash\left\{\Gamma_{B 0}\right\}} \sqrt{p_{B}} d_{B D, n}^{-a / 2} \mathbf{g}_{0 n} \mathbf{u}_{n} x_{B n} .
\end{aligned}
$$

Let $\mathbf{G}_{n}=\left[\mathbf{g}_{1 n}^{T} \mathbf{g}_{2 n}^{T} \cdots \mathbf{g}_{L n}^{T}\right], L \leq N-1 . \mathbf{g}_{L n}$ is the channel between the BS and the Lth DUE inside a circle of radius $r_{d}$ centered around the BS. Consider $L=\operatorname{round}(E[l])=$ $\operatorname{round}\left(\pi \lambda_{B} r_{d}^{2}\right)$, where $E[l]=\pi \lambda_{B} r_{d}^{2}$ denotes the DUEs number in circle of $r_{d}$ centered around the BS, and round(.) is round function. $\mathbf{u}_{n}$ lies in the null space of $\mathbf{G}_{n}$ to null the interference towards the $L$ DUEs and choose such that it maximizes the signal power $\left|\mathbf{q}_{n n} \mathbf{u}_{n}\right|^{2}$. From [17], $\mathbf{u}_{n}=$ $\mathbf{q}_{n n}^{H} \mathbf{S} \mathbf{S}^{H} /\left|\mathbf{q}_{n n}^{H} \mathbf{S S}{ }^{H}\right|$, where $\mathbf{S} \in \mathbb{C}^{N \times N-L}$ is the orthonormal basis of the null space of $\mathbf{G}_{n}$. The signal to interference ratio (SIR) in $R_{C 0}$ based on PZF strategy is

$$
\operatorname{SIR}_{C_{\mathrm{PZF}}}=\frac{P_{B} d_{B C}^{-a}\left|\mathbf{q}_{00} \mathbf{u}_{0}\right|^{2}}{I_{C_{\mathrm{PZF}}}}
$$

$I_{C_{\mathrm{PZF}}}$ denotes the total interference. One has

$$
\begin{aligned}
I_{C_{\mathrm{PZF}}}= & \sum_{n: \Gamma_{B n} \in \phi_{B} \backslash\left\{\Gamma_{B 0}\right\}} p_{B} d_{B C, n}^{-a}\left|\mathbf{q}_{0 n} \mathbf{u}_{n}\right|^{2} \\
& +\sum_{n: \Gamma_{D n} \in \phi_{D}} p_{D} d_{D C, n}^{-a}\left|f_{0 n}\right|^{2} .
\end{aligned}
$$

The signal to interference ratio (SIR) in $R_{D 0}$ based on PZF strategy is

$$
\operatorname{SIR}_{D_{\mathrm{PZF}}}=\frac{p_{D} d_{D D}^{-a}\left|h_{00}\right|^{2}}{I_{D_{\mathrm{PZF}}}}
$$

where $I_{D_{\mathrm{PZF}}}$ denotes the total interference and $I_{D_{\mathrm{PZF}, 1}}$ denotes the interference from BSs. $I_{D_{\mathrm{PZF}, 2}}$ denotes the interference from DUEs. Consider

$$
\begin{aligned}
I_{D_{\mathrm{PZF}}}= & \underbrace{\sum_{n: \Gamma_{D n} \in \phi_{D} \backslash\left\{\Gamma_{D 0}\right\}} p_{D} d_{D D, n}^{-a} h_{0 n} x_{D n}}_{I_{D_{\mathrm{PZF}, 1}}} \\
& +\underbrace{\sum_{n: \Gamma_{B n} \in \phi_{B} \backslash\left\{\Gamma_{B 0}\right\}} p_{B} d_{B D, n}^{-a}\left|\mathbf{g}_{0 n} \mathbf{u}_{n}\right|^{2}}_{I_{D_{\mathrm{PZF}, 2}}} .
\end{aligned}
$$

At the typical DUE receiver, the sum function subscript $\left(n: \Gamma_{B n} \in \phi_{B} \backslash\left\{\Gamma_{B 0}\right\}\right)$ in $I_{D_{\mathrm{PZF}, 2}}$ with PZF strategy is different from the subscript $\left(n: \Gamma_{B n} \in \phi_{B}\right)$ in $I_{D_{\mathrm{BF}, 2}}$ with BF strategy. $\left\{\Gamma_{B 0}\right\}$ denotes the BS which cancels the interference toward the typical DUE by PZF strategy. The typical CUE signal power is $\left|\mathbf{q}_{00} \mathbf{u}_{0}\right|^{2} \sim \chi^{2}(2(N-L))$ with PZF strategy. It is different from the typical CUE signal power $\left|\mathbf{q}_{00} \mathbf{u}_{0}\right|^{2} \sim$ $\chi^{2}(2 N)$ with BF strategy. $L$ is the degrees of freedom to null $L$ DUE interferences. The D2D and cellular network success probability with PZF strategy are given in the following theorem.
Theorem 3. For the cellular network underlay with D2D communication, the cellular BSs are configured with $N$ antennas for PZF strategy. The cellular and D2D success probability are given by

$$
\begin{aligned}
P_{S u c, D_{P Z F}}\left(\beta_{D}\right) & \\
=\exp (- & \lambda_{D} \frac{\pi}{\sin c(2 / a)}\left(\beta_{D}\right)^{2 / a} d_{D D}^{2} \\
& -\frac{2}{a-2} \pi \lambda_{B} r_{D}^{2-a} \frac{p_{B}}{p_{D}} d_{D D}^{a} \beta_{D 2} \\
& \left.\times F_{1}\left(1,1-\frac{2}{a} ; 2-\frac{2}{a} ;-\beta_{D} d_{D D}^{a} \frac{p_{B}}{p_{D}} r_{D}^{-a}\right)\right) \\
P_{S u c, C_{P Z F}} & \frac{\pi \lambda_{B}}{A_{0}}+\sum_{k=1}^{N-L-1} \sum_{j=1}^{k} \frac{(-1)^{k}}{k !} \pi \lambda_{B} A_{j}^{k} \frac{\Gamma(j+1)}{\left(A_{0}\right)^{j+1}}
\end{aligned}
$$

Proof. See Appendix C.

Theorem 4. For the cellular network underlay with D2D communication, the cellular BS is configured with $N$ antennas for PZF strategy. The cellular and D2D average achievable rate in a shared subchannel are given by

$$
\begin{aligned}
& R_{D_{P Z F}} \\
& =E\left[\log \left(1+\xi_{D_{P Z F}}\right)\right] \\
& =\int_{z=0}^{\infty} \frac{1-F_{\xi}(z)}{1+z} d z \\
& =\int_{z=0}^{\infty}\left(\operatorname { e x p } \left(-\lambda_{D} \frac{\pi}{\sin c(2 / a)}(z)^{2 / a} d_{D D}^{2}\right.\right. \\
& -\frac{2}{a-2} \pi \lambda_{B} r_{D}^{2-a} \frac{p_{B}}{p_{D}} d_{D D}^{a} z \\
& \left.\cdot{ }_{2} F_{1}\left(1,1-\frac{2}{a} ; 2-\frac{2}{a} ;-z d_{D D}^{a} \frac{p_{B}}{p_{D}} r_{D}^{-a}\right)\right) \\
& \left.\cdot(1+z)^{-1}\right) d z \\
& R_{C_{P Z F}}=E\left[\log \left(1+\xi_{C_{P Z F}}\right)\right] \\
& =\int_{0}^{\infty} \int_{z=0}^{\infty} \frac{\sum_{k=0}^{N-L-1}(1 / k !)(-z)^{k} Q(z, k)}{1+z} \\
& \cdot 2 \pi \lambda_{B} r \exp \left(-\pi \lambda_{B} r^{2}\right) d z d r .
\end{aligned}
$$

Proof. The proof is similar to that of Theorem 2.

Area spectral efficiency (ASE) is defined as the product of the unconditioned success probability and the maximum sum rate (in bps/Hz) that can be sent per unit area [13]. In order to maximize the ASE, we set a proper $r_{d}$ to get $L$. $L$ is the number of the canceled DUE interferences, which 
is also the degrees of freedom to null DUE interferences. So the BS transmission degrees of freedom can be allocated effectively between CUE beamforming and DUE interference cancellation. Consider

$$
\begin{aligned}
T_{D_{\mathrm{PZF}}=} & \lambda_{D} \log _{2}\left(1+\beta_{D}\right) P_{\mathrm{Suc}, D_{\mathrm{PZF}}}\left(\beta_{D}\right) \\
= & \lambda_{D} \log _{2}\left(1+\beta_{D}\right) \\
& \cdot \exp \left(-\lambda_{D} \frac{\pi}{\sin c(2 / a)}\left(\beta_{D}\right)^{2 / a} d_{D D}^{2}\right. \\
& -\frac{2}{a-2} \pi \lambda_{B} r_{D}^{2-a} \frac{p_{B}}{p_{D}} d_{D D}^{a} \beta_{D} \\
T_{C_{\mathrm{PZF}}=} & \lambda_{B} \log _{2}\left(1+\beta_{C}\right) P_{\mathrm{Suc}, C_{\mathrm{PZF}}}\left(\beta_{C}\right) \\
= & \lambda_{B} \log _{2}\left(1+\beta_{C}\right) \\
& \quad\left(\frac{\pi \lambda_{B}}{A_{0}}+\sum_{k=1}^{N-L-1} \sum_{j=1}^{k} \frac{(-1)^{k}}{k !} \pi \lambda_{B} A_{j}^{k} \frac{2}{\left(A_{0}\right)^{j+1}}\right) .
\end{aligned}
$$

The total ASE is

$$
\begin{aligned}
T_{\mathrm{PZF}}= & T_{D_{\mathrm{PZF}}}+T_{C_{\mathrm{PZF}}}=\lambda_{D} \log _{2}\left(1+\beta_{D}\right) \\
& \times \exp \left(-\lambda_{D} \frac{\pi}{\sin c(2 / a)}\left(\beta_{D}\right)^{2 / a} d_{D D}^{2}\right. \\
& -\frac{2}{a-2} \pi \lambda_{B} r_{D}^{2-a} \frac{p_{B}}{p_{D}} d_{D D}^{a} \beta_{D} \\
& \left.+\lambda_{B} F_{1}\left(1,1-\frac{2}{a} ; 2-\frac{2}{a} ;-\beta_{D} d_{D D}^{a} \frac{p_{B}}{p_{D}} r_{D}^{-a}\right)\right) \\
& \cdot\left(\frac{\pi \lambda_{B}}{A_{0}}+\sum_{k=1}^{N-L-1} \sum_{j=1}^{k} \frac{(-1)^{k}}{k !} \pi \lambda_{B} A_{j}^{k} \frac{\Gamma(j+1)}{\left(A_{0}\right)^{j+1}}\right) .
\end{aligned}
$$

In order to maximize the total ASE, it is difficult to get the close-form expression of optimal $r_{d}$. In this paper, we simulate the relation between ASE and $r_{d}$ and get the optimal $r_{d}$.

\section{Simulation Results and Discussion}

In this section, we present Monte Carlo simulations to evaluate the performance of $\mathrm{BF}$ and PZF strategies and discuss the relation between ASE and $r_{d}$ in this large random D2D underlaid cellular network. The simulated BS and DUE lie in a two-dimensional plane with independent Poisson processes. The default parameters are listed in Table 1 [10] unless otherwise stated. The analysis results developed in previous sections are validated with Monte Carlo simulations.
TABLE 1: Parameter assumptions.

\begin{tabular}{lcc}
\hline Parameter & Meaning & Default value \\
\hline$N$ & Number of BS antennas & $1,2,4,6$ \\
$V$ & Number of subchannels & 1000 \\
$\alpha$ & Pathloss exponent & 4 \\
$\lambda_{B}$ & Intensity of BS & $6 \times 10^{-6} / \mathrm{m}^{2}$ \\
$\lambda_{D}$ & Intensity of DUE & $2.4 \times 10^{-5} / \mathrm{m}^{2}$ \\
$\lambda_{U}$ & Intensity of CUE & $6 \times 10^{-4} / \mathrm{m}^{2}$ \\
$P_{C}$ & Transmission power of & $43 \mathrm{dBm}$ \\
$P_{D}$ & BSs \\
$\beta_{C}$ & $\begin{array}{c}\text { Transmission power of } \\
\text { DUE transmitter }\end{array}$ & $23 \mathrm{dBm}$ \\
$\beta_{D}$ & $\begin{array}{c}\text { SIR threshold of the } \\
\text { cellular network }\end{array}$ & $0 \mathrm{~dB}$ \\
$d_{D D}$ & $\begin{array}{c}\text { SIR threshold of the } \\
\text { cellular network } \\
\text { Distance between a D2D } \\
X_{C}\end{array}$ & $\begin{array}{c}\text { pair } \\
\text { Maximum transmit } \\
\text { range of CUE }\end{array}$ \\
\hline
\end{tabular}

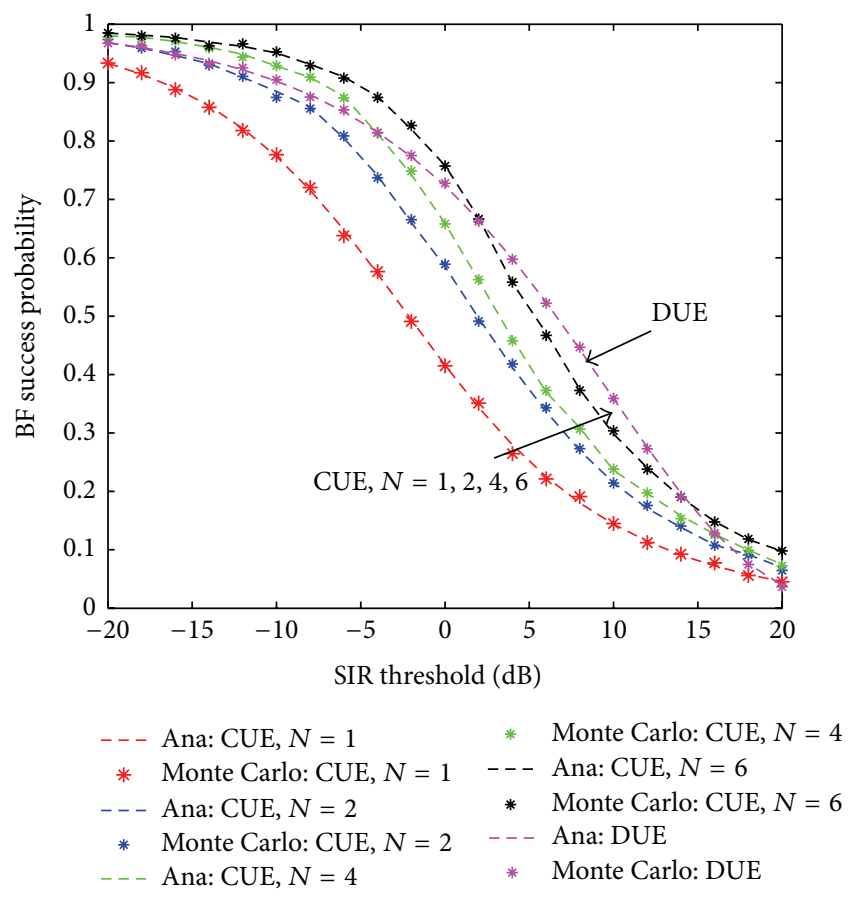

FIGURE 2: Cellular and D2D communication success probability in BF strategy.

Moreover, the analysis is performed to investigate the success probability as well as average achievable rate and illustrate the impact of the optimal $r_{d}$ to system ASE. The simulation is run for 10000 times and the average was taken.

Figure 2 shows the analytical and simulation success probability versus the SIR threshold in BF strategy. From the figures we can see the analytical model fits the simulation results fairly well and thus can conclude that our analysis is 


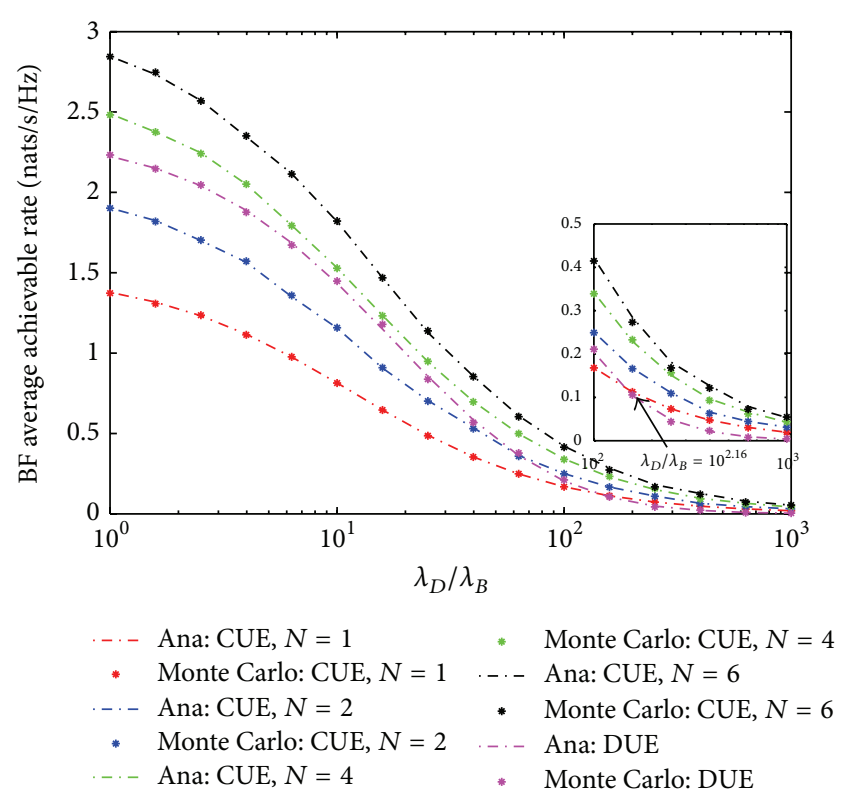

Figure 3: Average achievable rate for cellular and D2D communication in BF strategy.

well validated. The success probability of CUE in deployed multiantennas network is higher than single antenna case. In addition, the more antennas BS has the more dofs CUE get, but the change of BS antennas number makes no difference to success probability of DUE. This is because all the BS transmitter dofs are allocated to CUE in BF strategy.

Figure 3 validates the analysis results of average rate for both the cellular network and D2D communication in BF strategy. In addition, it illustrates how the average achievable rate is impacted as the increase of $\lambda_{D}$ with a fixed $\lambda_{B}$. From Figure 3, we observe the rate performances are severely degraded for all scenarios when the intensity ratio rises up to $10^{3}$. When the intensity ratio $\lambda_{D} / \lambda_{B}$ is a lower value, such as $10^{\circ}$, the average rate of DUE is higher than the average rate of CUE, but as the intensity ratio reaches $10^{2.16}$, we find that the average rate of DUE is lower than the average rate of CUE from the enlarged part figure. This is because the rate gain caused by the increase of DUE intensity is lesser than the rate degradation caused by the increase of many DUE interferences at the moment.

Figure 4 shows the analytical and simulation success probability versus the SIR threshold in PZF strategy. From Figure 4 , we find that the more dofs user get the higher success probability is.

Figure 5 validates the analysis results of average rate for both the cellular network and D2D communication in PZF strategy and compares the rate performance of two transmission strategies. When the BS deployed six antennas, the BS allocates 2 or 4 dofs to cancel the DUE interference in PZF strategy; then the BS allocates 4 or 2 dofs to increase the diversity gain of CUE accordingly. In BF strategy, all BS transmission dofs are allocated to their association CUE, and no dof is allocated to DUE.
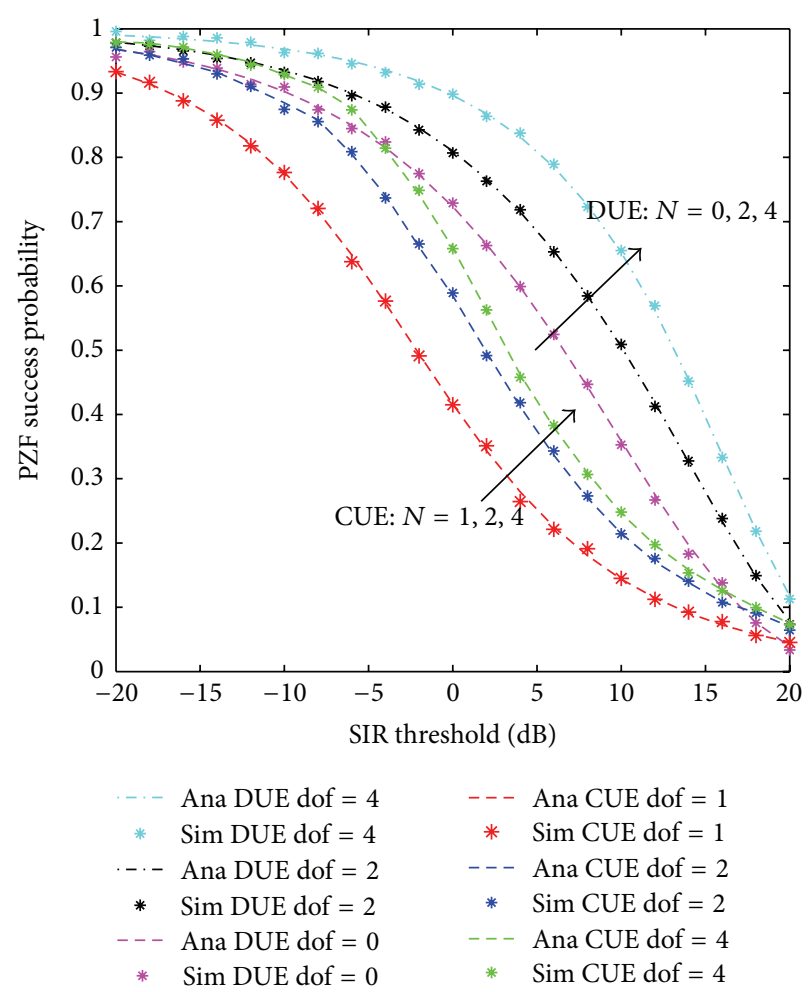

FIGURE 4: Cellular and D2D communication success probability for difference $N$ in PZF strategy. DUE dof = 0124 denotes 0, 1, 2, 4 dofs are allocated to DUE for interference cancellation, respectively. CUE dof = 0124 denotes $0,1,2,4$ dofs are allocated to CUE for increasing the diversity gain, respectively.

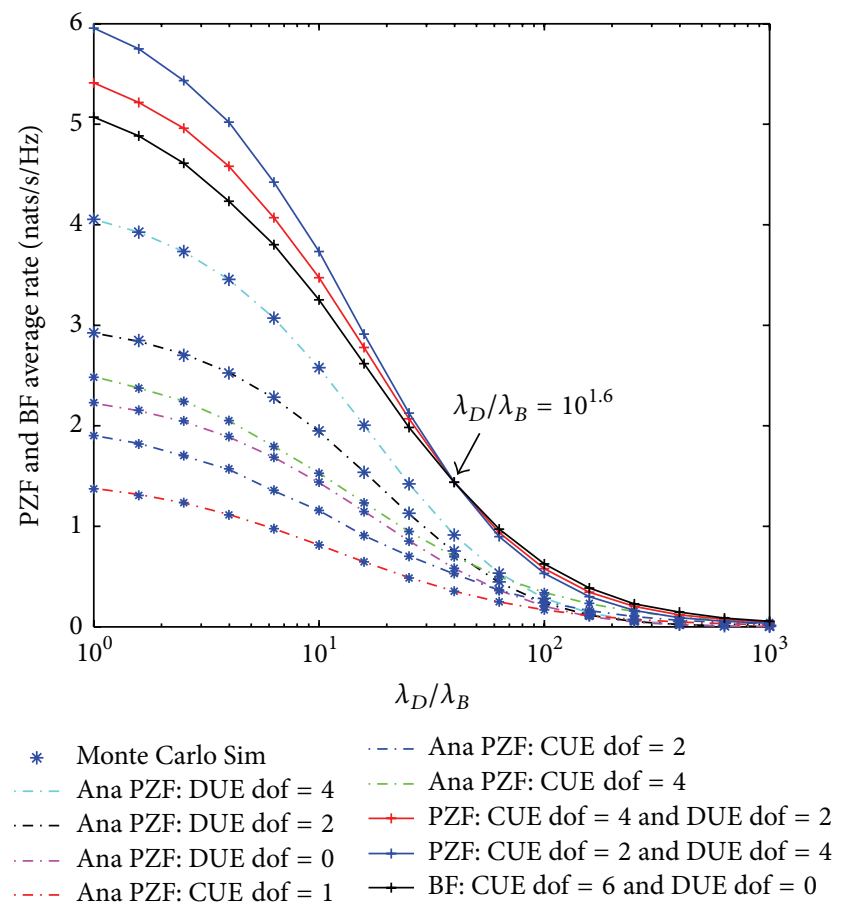

Figure 5: Average rate in PZF and BF strategies. CUE dof $=2,4$ and DUE dof $=4,2$ denote total simulation average rates include the cellular network and D2D communication in PZF strategy. 


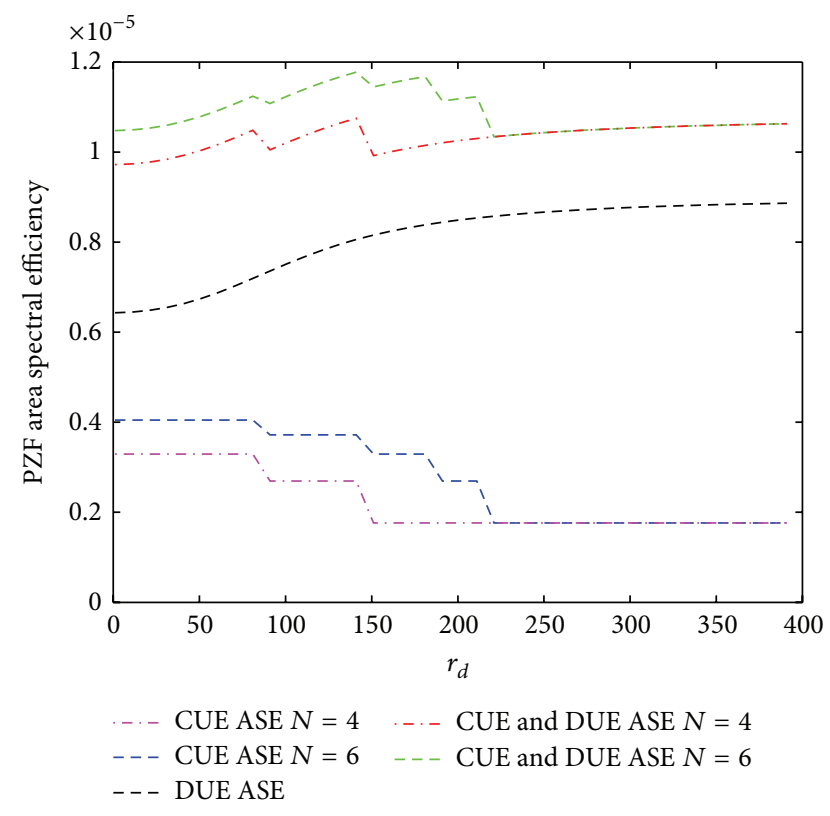

Figure 6: ASE with PZF strategy.

From Figure 5, we observe that when the intensity ratio is $\lambda_{D} / \lambda_{B}<10^{1.6}$, the average achievable total rate with PZF strategy is higher than the total rate with $\mathrm{BF}$ strategy. In order to maximize the total rate, we should allocate more dofs to DUE, but intensity ratio $\lambda_{D} / \lambda_{B} \geq 10^{1.6}$; the average achievable total rate with PZF strategy is lower than the total rate with $\mathrm{BF}$ strategy. This is because when the BS transmission dofs and CUE intensity are fixed, the rate gain due to the increase of DUE intensity is less than the rate degradation due to the increase of many DUE interferences.

Figure 6 shows the relation between the ASE and $r_{d}$. SIR threshold of the cellular network and D2D communications set to be $-5 \mathrm{~dB}$. From the figure, we see that when $r_{d}$ increases, the ASE of CUE decreases and the ASE of DUE increases. This is due to that fact that as $r_{d}$ increases, more dofs are allocated to DUE for interference cancelation, and less dofs are allocated to CUE for increasing the diversity gain. In addition, there exists an optimal $r_{d}$ to maximize the total ASE.

\section{Conclusion}

This paper analyzes the performance of the hybrid network of cellular and D2D communication with stochastic geometry theory. The analytical expressions of success probability and average rate are derived in BF and PZF transmission strategies, and the relation between ASE and interference cancellation radius is gotten. Simulation results show that the expressions can provide sufficient precision to evaluate the systems performance. In future study, we can consider the analysis of a hybrid network that the BS and CUEs/DUEs are both configured with multiple antennas by different precoding designs. These studies lay a theoretical foundation for network planning and base station deployment in hybrid network of cellular and D2D communication.

\section{Appendices}

\section{A. Proof of Theorem 1}

(1) Derivation of DUE success probability in BF strategy is as follows:

$$
\begin{aligned}
P_{\text {Suc. } D_{\mathrm{BF}}}\left(\beta_{D}\right) & =P\left\{\frac{p_{D} d_{D D}^{-a}\left|h_{00}\right|^{2}}{I_{D_{\mathrm{BF}}}}>\beta_{D}\right\} \\
& =P\left\{\left|h_{00}\right|^{2}>\beta_{D}\left(\widetilde{I}_{D_{\mathrm{BF}, 1}}+\widetilde{I}_{D_{\mathrm{BF}, 2}}\right)\right\} \\
& =L_{\widetilde{I}_{D_{\mathrm{BF}, 1}}}\left(\beta_{D}\right) L_{\widetilde{I}_{D_{\mathrm{BF}, 2}}}\left(\beta_{D}\right) .
\end{aligned}
$$

$L_{\widetilde{I}_{D_{\mathrm{PZF}}, 1}}\left(\beta_{D}\right)$ is the Laplace transform of the interference from other D2D transmitters and $L_{\widetilde{I}_{D_{\mathrm{PZF}, 2}}}\left(\beta_{D}\right)$ is the Laplace transform of the interference from other BS transmitters. One has

$$
\begin{aligned}
& L_{\widetilde{I}_{D_{\mathrm{BF}, 1}}}(s)=E\left[e^{-s \sum_{n: \Gamma_{D n} \in \phi_{D} \backslash\left\{\Gamma_{D 0}\right\}}\left(d_{D D, n}^{-a}\left|h_{0 n}\right|^{2} / d_{D D}^{-a}\right)}\right] \\
&=E^{! 0}\left[e^{-s \sum_{n: \Gamma_{D n} \in \phi_{D}}\left(d_{D D, n}^{-a}\left|h_{0 n}\right|^{2} / d_{D D}^{-a}\right)}\right] \\
&=e^{-2 \pi \lambda_{D} d_{D D}^{2} \int_{0}^{\infty}\left(1-E\left(\exp \left(-s r^{-a}\left|h_{0 n}\right|^{2}\right) r d r\right)\right)} \\
&=\exp \left(-\pi \lambda_{D} c(a) d_{D D}^{2} s^{2 / a}\right) \\
& L_{\widetilde{I}_{D_{\mathrm{BF}, 2}}(s)=} \exp \left(-\pi \lambda_{B} c(a) d_{D D}^{2}\left(\frac{p_{B}}{p_{D}}\right)^{2 / a} s^{2 / a}\right)
\end{aligned}
$$

where (A.2) follows the Campbells theorem as [5, 10, 20]. One has $c(a)=(2 \pi / a) / \sin (2 \pi / a)$. Substituting (A.2) and (A.3) into (A.1) yields the desired result in (6).

(2) Derivation of CUE success probability in BF strategy is as follows.

We rewrite SIR in $R_{C 0}$ based on BF strategy:

$$
\begin{gathered}
\operatorname{SIR}_{C_{\mathrm{BF}}}=\frac{P_{B} d_{B C}^{-a}\left|\mathbf{q}_{00} \mathbf{u}_{0}\right|^{2}}{I_{C_{\mathrm{BF}}}}, \\
I_{C_{\mathrm{BF}}}=\underbrace{\underbrace{\sum_{I_{\mathrm{BF}, 2}}}_{\sum_{n: \Gamma_{D n} \in \phi_{D}} p_{D} d_{D C, n}^{-a}\left|f_{0 n}\right|^{2}}}_{I_{C_{\mathrm{BF}, 1}} \sum_{\Gamma_{B n} \in \phi_{B} \backslash\left\{\Gamma_{B 0}\right\}} p_{B} d_{B C, n}^{-a}\left|\mathbf{q}_{0 n} \mathbf{u}_{n}\right|^{2}}
\end{gathered}
$$

where $\widetilde{I}_{C_{\mathrm{BF}, 1}}$ and $\widetilde{I}_{C_{\mathrm{BF}, 2}}$ are simple transform of $I_{C_{\mathrm{BF}, 1}}$ and $I_{C_{\mathrm{BF}, 2}}$ by normalizing the desired signal power, respectively:

$$
\begin{aligned}
& \widetilde{I}_{C_{B F, 1}}=\sum_{n: \Gamma_{B n} \in \phi_{B} \backslash\left\{\Gamma_{B 0}\right\}} \frac{d_{B C, n}^{-a}\left|\mathbf{q}_{0 n} \mathbf{u}_{n}\right|^{2}}{d_{B C}^{-a}}, \\
& \tilde{I}_{C_{B F, 2}}=\sum_{n: \Gamma_{D n} \in \phi_{D}} \frac{d_{D C, n}^{-a}\left|f_{0 n}\right|^{2}}{d_{B C}^{-a} p_{D} / p_{B}} .
\end{aligned}
$$


When $d_{B C}$ is known,

$$
\begin{aligned}
& L_{\widetilde{I}_{C_{\mathrm{BF}, 1}}}(s) \approx \exp \left(-\pi \lambda_{B} c(a) d_{B C}^{2} s^{2 / a}\right), \\
& L_{\widetilde{I}_{C_{\mathrm{BF}, 2}}}(s)=\exp \left(-\pi \lambda_{D} c(a) d_{B C}^{2}\left(\frac{p_{D}}{p_{B}}\right)^{2 / a} s^{2 / a}\right), \\
& C_{d 2}=\pi c(a) d_{B C}^{2}\left(\lambda_{B}+\lambda_{D}\left(\frac{p_{D}}{p_{B}}\right)^{2 / a}\right), \\
& L_{\widetilde{I}_{C_{\mathrm{BF}}}}(s)=L_{\widetilde{I}_{\mathrm{CF}_{\mathrm{BF}, 1}}}(s) L_{\widetilde{I}_{\mathrm{CBF}, 2}}(s)=\exp \left(C_{d 2} s^{2 / a}\right) .
\end{aligned}
$$

Let the signal power $S_{C}=\left|\mathbf{q}_{00} \mathbf{u}_{0}\right|^{2}$ and the complementary cumulative distribution function (CCDF) of $S_{C}$ is given by

$$
F_{S_{c}}(t)=e^{-t} \sum_{k=0}^{N-1} \frac{t^{k}}{k !}
$$

The probability density function (PDF) of the distance $d_{B C}$ is given by

$$
f_{d_{B C}}(r)=2 \pi \lambda_{B} r \exp \left(-\pi \lambda_{B} r^{2}\right)
$$

CUE success probability in BF strategy can be derived as

$$
\begin{aligned}
P_{\text {Suc. } C_{\mathrm{BF}}}\left(\beta_{C}\right) & =\left.E_{d_{B C}}\left[P\left(\operatorname{SIR}_{C_{\mathrm{BF}}}>\beta_{C} \mid d_{B C}\right)\right]\right|_{s=\beta_{C}} \\
& =\int_{0}^{\infty} P\left(\operatorname{SIR}_{C_{\mathrm{BF}}}>\beta_{C} \mid d_{B C}\right) f_{d_{B C}}(r) d r \\
& =\int_{0}^{\infty} P\left\{\frac{p_{B} r^{-a}\left|\mathbf{q}_{00} \mathbf{u}_{0}\right|^{2}}{I_{C_{\mathrm{BF}}}}>\beta_{C}\right\} f_{d_{B C}}(r) d r \\
& =\left.\int_{0}^{\infty} \int_{0}^{\infty} F_{S_{c}}(s t) f_{\widetilde{I}_{C_{\mathrm{BF}}}}(t) f_{d_{B C}}(r) d t d r\right|_{s=\beta_{C}} .
\end{aligned}
$$

In the following, we will calculate the double integral

$$
\begin{aligned}
& \int_{0}^{\infty} \int_{0}^{\infty} F_{S_{c}}(s t) f_{\widetilde{I}_{C_{B F}}}(t) f_{d_{B C}}(r) d t d r \\
& =\int_{0}^{\infty} \int_{0}^{\infty} e^{-s t} \sum_{k=0}^{N-1} \frac{(s t)^{k}}{k !} f_{\widetilde{I}_{C_{\mathrm{BF}}}}(t) d t f_{d_{B C}}(r) d r \\
& =\int_{0}^{\infty} \sum_{k=0}^{N-1} \frac{1}{k !} s^{k}\left(\int_{0}^{\infty} e^{-t} t^{k} f_{\widetilde{I}_{C_{\mathrm{BF}}}}(t) d t\right) f_{d_{B C}}(r) d r \\
& \stackrel{\mathrm{a}}{=} \int_{0}^{\infty} \sum_{k=0}^{N-1} \frac{1}{k !} s^{n} L\left\{t^{k} f_{\widetilde{I}_{C_{\mathrm{BF}}}}(t)\right\}(s) f_{d_{B C}}(r) d r \\
& =\int_{0}^{\infty} \sum_{k=0}^{N-1} \frac{1}{k !}(-s)^{k} \frac{d^{k} L_{\widetilde{I}_{C_{\mathrm{BF}}}}(s)}{d s^{k}} f_{d_{B C}}(r) d r,
\end{aligned}
$$

where step (a) is achieved by utilizing the property of Laplace transform

$$
t^{n} f(t) \longleftrightarrow(-1)^{n} \frac{d^{n}}{d s^{n}} L[f(t)](s)
$$

Let $C_{d 2}^{\prime}=-\pi c(a)\left(\lambda_{B}+\lambda_{D}\left(p_{D} / p_{B}\right)^{2 / a}\right)$ and $Q(s, k)=$ $d^{k} L_{\widetilde{I}_{\mathrm{C}_{\mathrm{BF}}}}(s) / d s^{k}$. Employing the $n$th derivation of the composite function and after some algebra, we can obtain $Q(s, k)$ as [10]

$$
\begin{aligned}
Q & (s, k) \\
& =\frac{d L_{\widetilde{I}_{C_{\mathrm{BF}}}}(s)}{d s^{k}} \\
& = \begin{cases}\exp \left(-C_{d 2} s^{2 / a}\right), & k=0 \\
\left(-\frac{1}{s}\right)^{k} \exp \left(-C_{d 2} s^{2 / a}\right) \sum_{j=1}^{k} \frac{\beta_{j}^{k}}{j !}\left(C_{d 2} s^{2 / a}\right)^{j}, & k \neq 0 .\end{cases}
\end{aligned}
$$

Substituting (A.12) into (A.10) and setting $a=4$ and $s=\beta_{C}$ yield the desired CUE success probability:

$$
\begin{aligned}
& P_{\text {Suc. } C_{\mathrm{BF}}} \beta_{C}=E_{d_{B C}}\left[P\left(\operatorname{SIR}_{C_{\mathrm{BF}}}>\beta_{C} \mid d_{B C}\right)\right] \\
& =\int_{0}^{\infty} P\left(\operatorname{SIR}_{C_{B F}}>\beta_{C} \mid d_{B C}\right) f_{d_{B C}}(r) d r \\
& =\int_{0}^{\infty} \sum_{k=0}^{N-1} \frac{1}{k !}(-s)^{k} Q(s, k) f_{d_{B C}}(r) d r \\
& =\int_{0}^{\infty} 2 \pi \lambda_{B} r \exp \left(-\left(C_{d 2}^{\prime} s^{2 / a}+\pi \lambda_{B}\right) r^{2}\right) d r \\
& +\int_{0}^{\infty} \frac{(-1)^{k}}{k !} 2 \pi \lambda_{B} r \exp \left(-\left(C_{d 2}^{\prime} s^{2 / a}+\pi \lambda_{B}\right) r^{2}\right) \\
& \cdot \sum_{k=1}^{N-1} \sum_{j=1}^{k} \frac{\beta_{j}^{k}}{j !}\left(C_{d 2}^{\prime} s^{2 / a}\right)^{j} r^{2 j} d r \\
& =\int_{0}^{\infty} 2 \pi \lambda_{B} r \exp \left(-A_{0} r^{2}\right) d r \\
& +\int_{0}^{\infty} \frac{(-1)^{k}}{k !} 2 \pi \lambda_{B} \sum_{k=1}^{N-1} \sum_{j=1}^{k} A_{j}^{k} \exp \left(-A_{0} r^{2}\right) r^{2 j+1} d r \\
& =\frac{\pi \lambda_{B}}{A_{0}}+\sum_{k=1}^{N-1} \sum_{j=1}^{k} \frac{(-1)^{k}}{k !} \pi \lambda_{B} A_{j}^{k} \frac{\Gamma(j+1)}{\left(A_{0}\right)^{j+1}},
\end{aligned}
$$

where $A_{0}$ and $A_{j}^{k}$ are denoted in (9) and (10). 


\section{B. Proof of Theorem 2}

(1) Derivation of average rate of CUE is as follows:

$$
\begin{gathered}
E[\log (1+\xi)] \\
=\int_{0}^{\infty} E\left[\log (1+\xi) \mid d_{B C}\right] f_{d_{B C}}(r) d r \\
=\int_{0}^{\infty} \int_{z=0}^{\infty} \frac{\sum_{k=0}^{N-1}(1 / k !)(-z)^{k} Q(z, k)}{1+z} 2 \pi \lambda_{B} r \\
\cdot \exp \left(-\pi \lambda_{B} r^{2}\right) d z d r .
\end{gathered}
$$

(2) Derivation of average rate of DUE is as follows:

$$
\begin{aligned}
R_{D_{\mathrm{BF}}} & =E\left[\log \left(1+\xi_{D_{\mathrm{BF}}}\right)\right] \\
& =\int_{z=0}^{\infty} \frac{1-F_{\xi}(z)}{1+z} d z \\
& =\int_{z=0}^{\infty} \frac{\exp \left(-C_{d 1} z^{2 / a}\right)}{1+z} d z \\
& =\Lambda\left(C_{d 1}\right) .
\end{aligned}
$$

\section{Proof of Theorem 3}

(1) Derivation of DUE success probability $P_{\text {Suc. } D_{\mathrm{PZF}}}\left(\beta_{D}\right)$ is as follows.

The signal to interference ratio $\operatorname{SIR}_{D_{\mathrm{PZF}}}$ in $R_{D 0}$ is denoted in (21). $I_{D_{\mathrm{PZF}, 1}}$ denotes the interference from BSs. $I_{D_{\mathrm{PZF}, 2}}$ denotes the interference from DUEs; $\widetilde{I}_{D_{\mathrm{PZF}, 1}}$ and $\widetilde{I}_{D_{\mathrm{PZF}, 2}}$ are simple transform of $I_{D_{\mathrm{PZF}, 1}}$ and $I_{D_{\mathrm{PZF}, 2}}$ by dividing the desired signal power, respectively:

$$
\begin{aligned}
& \widetilde{I}_{D_{\mathrm{PZF}, 1}}=\sum_{n: \Gamma_{D n} \in \phi_{D} \backslash\left\{\Gamma_{D 0}\right\}} \frac{d_{D D, n}^{-a}\left|h_{0 n}\right|^{2}}{d_{D D}^{-a}}, \\
& \widetilde{I}_{D_{\mathrm{PZF}, 2}}=\sum_{n: \Gamma_{B n} \in \phi_{B} \backslash\left\{\Gamma_{B 0}\right\}} \frac{d_{B D, n}^{-a}\left|\mathbf{g}_{0 n} \mathbf{u}_{n}\right|^{2}}{d_{D D}^{-a} p_{D} / p_{B}} .
\end{aligned}
$$

The success transmission probability of DUE is

$$
\begin{aligned}
& P_{\text {Suc }, D_{\mathrm{PZF}}}\left(\beta_{D}\right) \\
& \quad=P\left(\operatorname{SIR}_{D_{\mathrm{PZF}}}>\beta_{D}\right) \\
& \quad=P\left(\frac{\left|h_{00}\right|^{2}}{\widetilde{I}_{D_{\mathrm{PZF}}}}>\beta_{D}\right) \\
& =E\left[\exp \left(-\beta_{D}\left(\widetilde{I}_{D_{\mathrm{PZF}, 1}}+\widetilde{I}_{D_{\mathrm{PZF}, 2}}\right)\right)\right] \\
& =L_{\widetilde{I}_{D_{\mathrm{PZF}, 1}}}\left(\beta_{D}\right) L_{\widetilde{I}_{\mathrm{DPZF}^{2},}}\left(\beta_{D}\right) .
\end{aligned}
$$

Equation (C.2) follows from $\left|h_{00}\right|^{2} \sim \exp (1)$ as $[1,10] . \widetilde{I}_{D_{\mathrm{PZF}}}=$ $\widetilde{I}_{D_{\mathrm{PZF}, 1}}+\widetilde{I}_{D_{\mathrm{PZF}, 2}}, L_{\widetilde{I}_{\mathrm{DPZF}_{1}}}\left(\beta_{D}\right)$ is the Laplace transform of the interference from other D2D transmitters, and $L_{\widetilde{I}_{D_{\mathrm{PZF}, 2}}}\left(\beta_{D}\right)$ is the Laplace transform of the interference from BS transmitters. Consider

$$
\begin{aligned}
& \left.L_{\widetilde{I}_{D_{\mathrm{PZF}, 1}}}(s)\right|_{s=\beta_{D}} \\
& =\left.\exp \left(-\pi \lambda_{D} c(a) d_{D D}^{2} s^{2 / a}\right)\right|_{s=\beta_{D}} \\
& =\exp \left(-\lambda_{D} \frac{\pi}{\sin c(2 / a)}\left(\beta_{D}\right)^{2 / a} d_{D D}^{2}\right) \text {, } \\
& L_{\widetilde{I}_{D_{\mathrm{PZF}, 2}}}(s) \\
& =E\left[\exp \left(-s \frac{p_{B}}{d_{D D}^{-a} p_{D}} r^{-a} W\right)\right] \\
& =\exp \left\{-2 \pi \lambda_{B} \int_{r_{D}}^{\infty}\left(1-\frac{1}{1+s p_{B} d_{D D}^{a} p_{D}^{-1} r^{-a}}\right) r d r\right\} \\
& =\exp \left\{-\frac{2}{a-2} \pi \lambda_{B} r_{D}^{2-a} \frac{p_{B}}{p_{D}} d_{D D}^{a} s\right. \\
& \left.\cdot{ }_{2} F_{1}\left(1,1-\frac{2}{a} ; 2-\frac{2}{a} ;-s \frac{p_{B}}{p_{D}} d_{D D}^{a} r_{D}^{-a}\right)\right\},
\end{aligned}
$$

where $W=\left|\mathbf{g}_{0 n} \mathbf{u}_{n}\right|^{2},{ }_{2} F_{1}(\cdot)$ is Gause hypergeometry function [1]. Substituting (C.3) into (C.2) yields the desired result in (23).

(2) Derivation of CUE success probability $P_{\text {Suc. } C_{\mathrm{PZF}}}\left(\beta_{C}\right)$ is as follows.

The typical CUE signal power is $\left|\mathbf{q}_{00} \mathbf{u}_{0}\right|^{2} \sim \chi^{2}(2(N-$ $L)$ ) with PZF strategy, and the typical CUE signal power $\left|\mathbf{q}_{00} \mathbf{u}_{0}\right|^{2} \sim \chi^{2}(2 N)$ with BF strategy. There is only difference in degree of freedom in two strategies. So we change the $N$ in (7) into $N-L$; then we get (24).

\section{Conflict of Interests}

The authors declare that there is no conflict of interests regarding the publication of this paper.

\section{Acknowledgments}

The authors gratefully acknowledge anonymous reviewers who read drafts and made many helpful suggestions. This study is supported by the Natural Science Foundation of China (no. 60872149 and no. 61471056), BUPT Youth Research and Innovation Plan (2014RC0103), and the National High Technology Research and Development Program of China (863 Program) (no. 2014AA01A701).

\section{References}

[1] X. Lin, J. G. Andrews, and A. Ghosh, "Spectrum sharing for device-to-device communication in cellular networks," IEEE Transactions on Wireless Communications, vol. 13, no. 12, pp. 6727-6740, 2014. 
[2] Z. Liu, T. Peng, H. Chen, and W. Wang, "Transmission capacity of D2D communication under heterogeneous networks with multi-bands," in Proceedings of the IEEE 77th Vehicular Technology Conference (VTC Spring '13), pp. 1-6, IEEE, June 2013.

[3] J. Liu, N. Kato, J. Ma, and N. Kadowaki, "Device-to-device communication in LTE-advanced networks: a survey," IEEE Communications Surveys \& Tutorials, 2014.

[4] S. Akoum and R. W. Heath, "Interference coordination: random clustering and adaptive limited feedback," IEEE Transactions on Signal Processing, vol. 61, no. 7, pp. 1822-1834, 2013.

[5] J. G. Andrews, F. Baccelli, and R. K. Ganti, "A tractable approach to coverage and rate in cellular networks," IEEE Transactions on Communications, vol. 59, no. 11, pp. 3122-3134, 2011.

[6] R. W. Heath, M. Kountouris, and B. Tianyang, "Modeling heterogeneous network interference using poisson point processes," IEEE Transactions on Signal Processing, vol. 61, no. 16, pp. 4114-4126, 2013.

[7] H. S. Dhillon, R. K. Ganti, F. Baccelli, and J. G. Andrews, "Modeling and analysis of K-tier downlink heterogeneous cellular networks," IEEE Journal on Selected Areas in Communications, vol. 30, no. 3, pp. 550-560, 2012.

[8] M. C. Erturk, S. Mukherjee, H. Ishii, and H. Arslan, "Distributions of transmit power and SINR in device-to-device networks," IEEE Communications Letters, vol. 17, no. 2, pp. 273276, 2013

[9] H. ElSawy, E. Hossain, and M.-S. Alouini, "Analytical modeling of mode selection and power control for underlay D2D communication in cellular networks," IEEE Transactions on Communications, vol. 62, no. 11, pp. 4147-4161, 2014.

[10] P. Mugen, L. Yuan, T. Q. S. Quek, and W. Chonggang, "Deviceto-device underlaid cellular networks under rician fading channels," IEEE Transactions on Wireless Communications, vol. 13, no. 8, pp. 4247-4259, 2014.

[11] H. S. Dhillon, M. Kountouris, and J. G. Andrews, "Downlink MIMO HetNets: modeling, ordering results and performance analysis," IEEE Transactions on Wireless Communications, vol. 12, no. 10, pp. 5208-5222, 2013.

[12] X. Zhang, L. Zhang, G. Lu, X. Chu, and Y. Zou, "Advanced MIMO techniques in the heterogeneous networks," International Journal of Antennas and Propagation, vol. 2014, Article ID 193954, 2 pages, 2014.

[13] M. Kountouris and J. G. Andrews, "Downlink SDMA with limited feedback in interference-limited wireless networks," IEEE Transactions on Wireless Communications, vol. 11, no. 8, pp. 2730-2741, 2012.

[14] A. Barbieri, P. Gaal, S. Geirhofer et al., "Coordinated downlink multi-point communications in heterogeneous cellular networks," in Proceedings of the Information Theory and Applications Workshop (ITA '12), pp. 7-16, February 2012.

[15] H. Kaibin, J. G. Andrews, G. Dongning, J. Heath, and R. A. Berry, "Spatial interference cancellation for multiantenna mobile ad hoc networks," IEEE Transactions on Information Theory, vol. 58, no. 3, pp. 1660-1676, 2012.

[16] Y. Li, B. Lin, C. Chen, J. Ye, and J. Choi, "Successive orthogonal beamforming for cooperative multi-point downlinks," IET Communications, vol. 7, no. 8, pp. 706-714, 2013.

[17] R. Vaze and R. W. Heath, "Transmission capacity of adhoc networks with multiple antennas using transmit stream adaptation and interference cancellation," IEEE Transactions on Information Theory, vol. 58, no. 2, pp. 780-792, 2012.
[18] R. Cai, P. C. Ching, and T. N. Davidson, "Tradeoff analysis of partial zero-forcing beamforming in large random spectrum sharing networks," in Proceedings of the IEEE 15th International Workshop on Signal Processing Advances in Wireless Communications (SPAWC '14), pp. 75-79, June 2014.

[19] S. Hari and Y. Wei, "Partial zero-forcing precoding for the interference channel with partially cooperating transmitters," in Proceedings of the IEEE International Symposium on Information Theory (ISIT '10), pp. 2283-2287, June 2010.

[20] H. Feng, H. Wang, X. Xu, and C. Xing, "A tractable model for device-to-device communication underlaying multi-cell cellular networks," in Proceedings of the IEEE International Conference on Communication Workshop (ICC '14), pp. 587-591, Sydney, Australia, June 2014. 

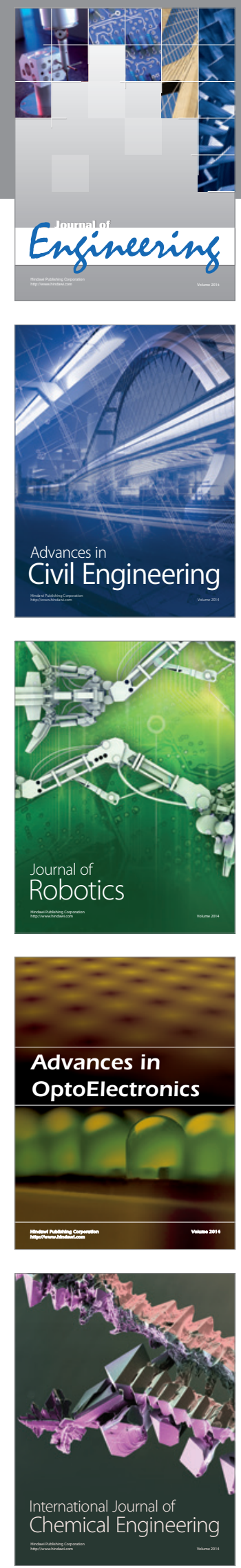

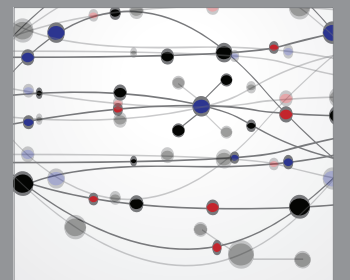

The Scientific World Journal
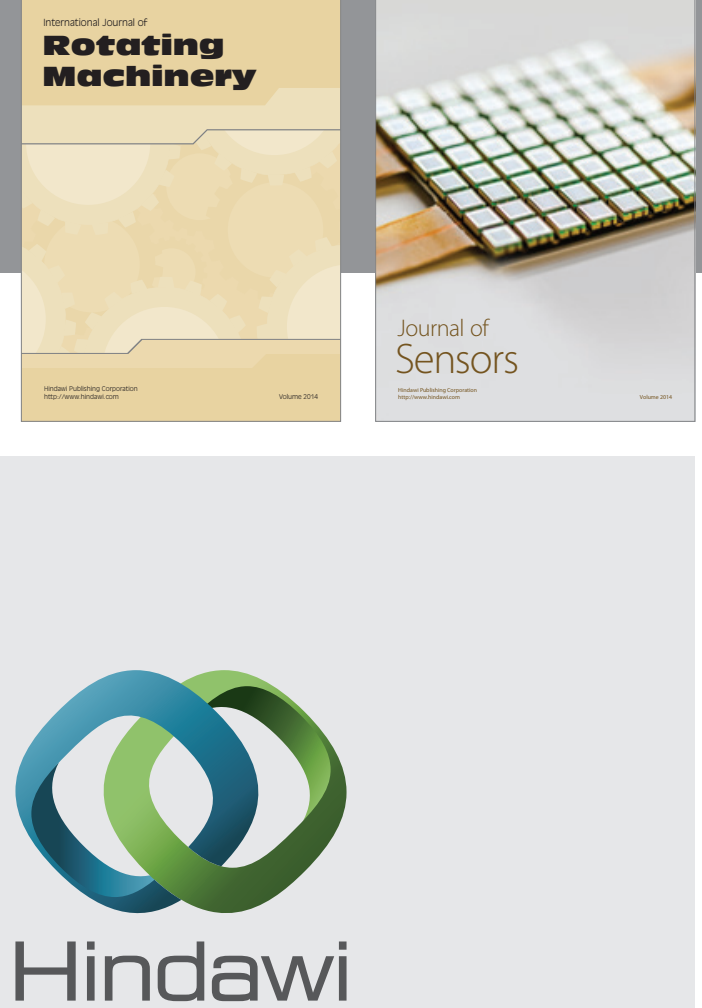

Submit your manuscripts at http://www.hindawi.com
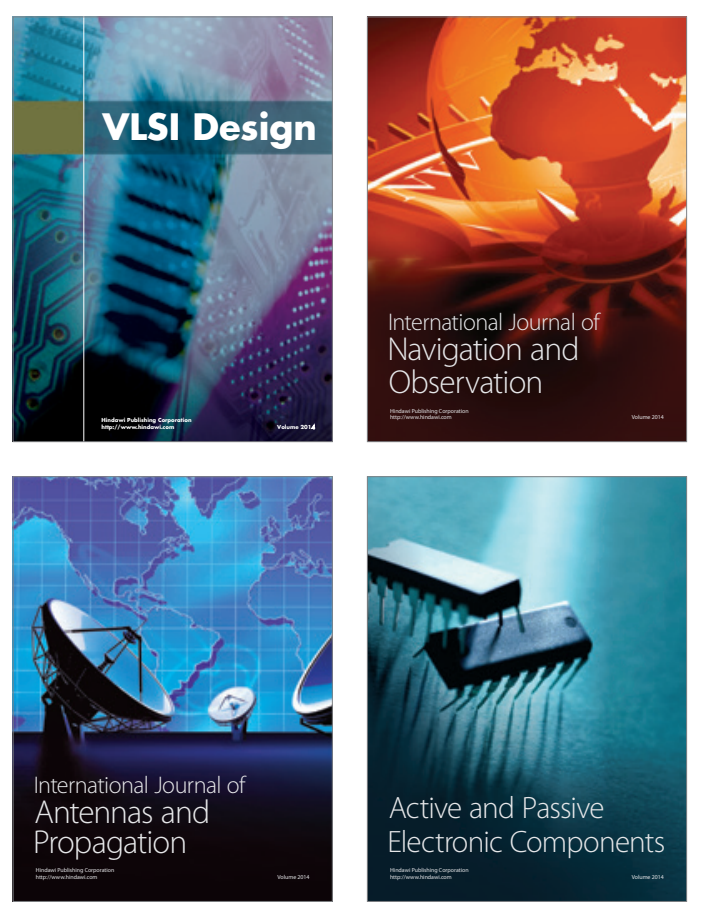
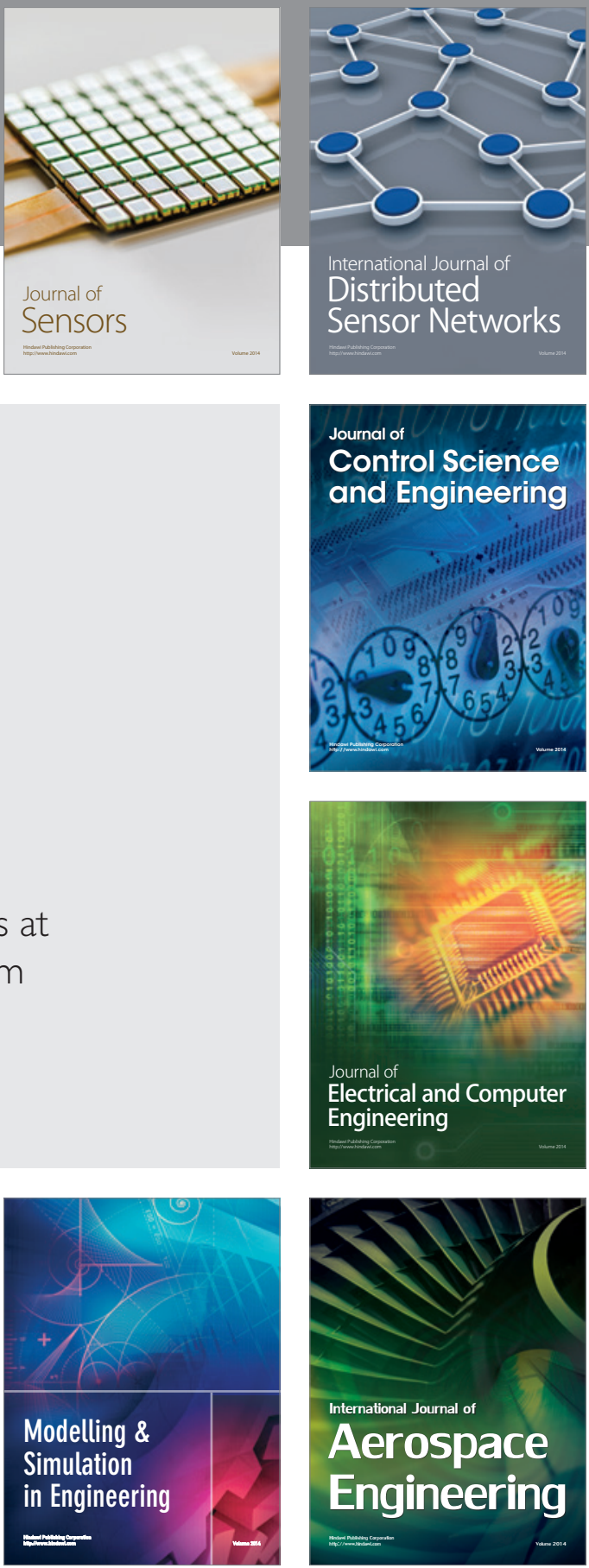

Journal of

Control Science

and Engineering
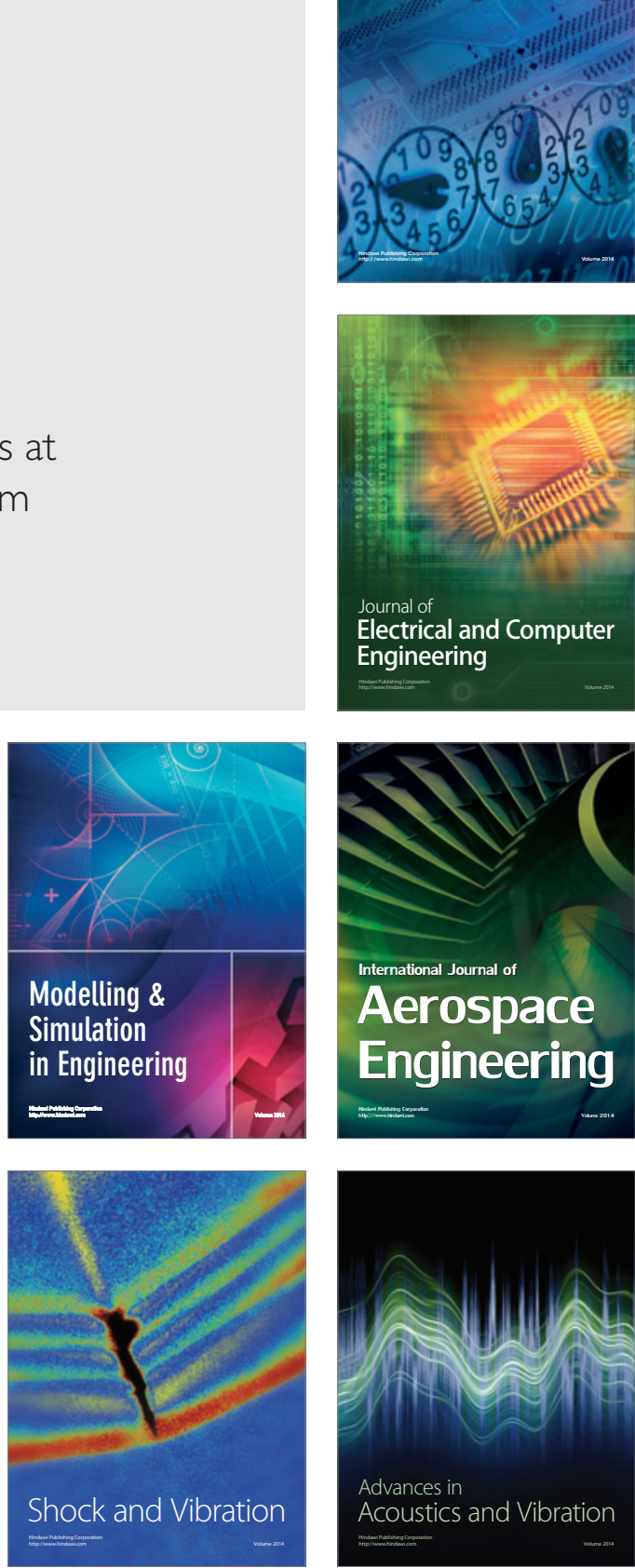